\title{
The Australian Square Kilometre Array Pathfinder
}

\section{Tobias Westmeier*}

CSIRO Astronomy and Space Science

E-mail: tobias.westmeier@csiro.au

\section{Simon Johnston}

CSIRO Astronomy and Space Science

E-mail: Simon.johnstonecsiro.au

The Australian Square Kilometre Array Pathfinder (ASKAP) is a next-generation synthesis radio telescope and a precursor of the Square Kilometre Array (SKA). It is located at the Murchison Radio-astronomy Observatory in Western Australia, which is characterised by very low levels of radio interference. When completed in 2013, ASKAP will consist of 36 parabolic antennas, each $12 \mathrm{~m}$ in diameter, with a maximum baseline of about $6 \mathrm{~km}$. Each antenna will be equipped with a phased array feed providing an instantaneous field of view of approximately 30 square degrees. Hence, ASKAP will be a fast survey instrument capable of mapping large areas on the sky with unprecedented speed and sensitivity.

During the first five years of operation, $75 \%$ of the available observing time will be dedicated to large Survey Science Projects which require more than 1500 hours of observing time and make use of ASKAP's unique capabilities and survey speed. In an open, international, and competitive process, ten Survey Science Projects were identified to work with CSIRO through the design study phase. These projects will address widely recognised astrophysical issues relevant to the SKA key science projects, including, for example, the evolution of galaxies, the role of magnetic fields in the universe, and the study of pulsars.

ISKAF2010 Science Meeting - ISKAF2010

June 10-14, 2010

Assen, the Netherlands

\footnotetext{
* Speaker.
} 

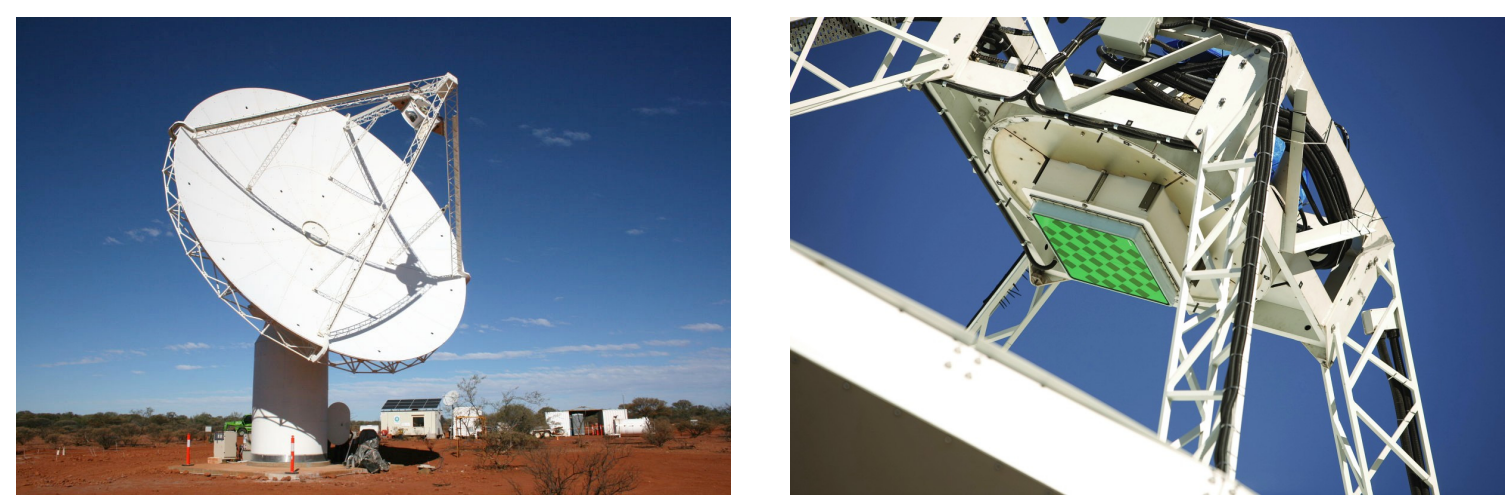

Figure 1: Left: The first ASKAP antenna, named "Diggidumble", was erected at the MRO site in Western Australia in late 2009 (photo courtesy of Simon Johnston, CSIRO). Right: A smaller prototype of the phased array feed was installed on the 12-m testbed antenna at Parkes for testing purposes (photo courtesy of David McClenaghan, CSIRO).

\section{Overview of ASKAP}

The Australian Square Kilometre Array Pathfinder (ASKAP) is a next-generation synthesis radio telescope being developed and built by CSIRO as part of the Australia Telescope National Facility [1]. It is one of the precursor telescopes of the Square Kilometre Array (SKA) [2] as part of Australia's and New Zealand's bid to host the SKA. ASKAP is located at the Murchison Radioastronomy Observatory (MRO) at approximately $26^{\circ} 42^{\prime} 15^{\prime \prime} \mathrm{S}$ and $116^{\circ} 39^{\prime} 32^{\prime \prime}$ E near Boolardy in Western Australia. The MRO also hosts the Murchison Widefield Array (MWA) [3] and constitutes the proposed core site for the SKA in Australia.

The main goals of ASKAP are

- to demonstrate and prototype technologies for the mid-frequency SKA, including field-ofview enhancement by phased array feeds on $12 \mathrm{~m}$-class parabolic reflectors,

- to carry out world-class, ground-breaking observations directly relevant to the SKA key science projects,

- to establish a site for radio astronomy in Western Australia where observations can be carried out free from the harmful effects of radio interference, and

- to establish a user community for the SKA.

An overview of the design goals of ASKAP is given in Table 1. ASKAP will consist of 36 antennas, each of which will have a diameter of $12 \mathrm{~m}$ (Fig. 1), resulting in a total collecting area of just over $4000 \mathrm{~m}^{2} .30$ antennas will be concentrated in a 2-km core array, with the remaining six antennas spread further out with a maximum baseline of about $6 \mathrm{~km}$ (Fig. 2); also see [4]), resulting in a maximum angular resolution of approximately $10^{\prime \prime}$ at a frequency of $1.4 \mathrm{GHz}$. A histogram of telescope baselines for the initial ASKAP configuration is shown in the right-hand panel of Fig. 2. The antenna configuration will provide excellent $u v$ coverage, even at declinations 


\begin{tabular}{lrl}
\hline Parameter & Value & Unit \\
\hline Number of antennas & 36 & \\
Antenna diameter & 12 & $\mathrm{~m}$ \\
Maximum baseline & 6 & $\mathrm{~km}$ \\
Angular resolution & 10 & $\mathrm{arcsec}$ \\
System temperature & 50 & $\mathrm{~K}$ \\
Sensitivity & 65 & $\mathrm{~m}^{2} / \mathrm{K}$ \\
Phased array feed elements & 192 & \\
Instantaneous field of view & 30 & $\mathrm{deg}^{2}$ \\
Frequency range & $0.7-1.8$ & $\mathrm{GHz}$ \\
Processed bandwidth & 300 & $\mathrm{MHz}$ \\
Number of spectral channels & 16384 & \\
\hline
\end{tabular}

Table 1: Overview of ASKAP design goals.

near $0^{\circ}$, resulting in low sidelobe levels of the synthesised beam and high image fidelity. The $u v$ coverage $^{1}$ for declinations of $-50^{\circ}$ and $-10^{\circ}$ is shown in Fig. B.

The design of ASKAP is unique among radio telescopes. Its antennas feature three-axis movement to keep the orientation of the aperture fixed with respect to the sky. This will allow high dynamic range imaging and polarisation measurements of high purity. Furthermore, ASKAP will be equipped with phased array feeds (Fig. 1) rather than single-pixel feeds, a development being pioneered by CSIRO in conjunction with colleagues in the Netherlands, Canada, the United Kingdom, and Germany. The phased array feed will provide an instantaneous field of view of about 30 square degrees, making ASKAP a dedicated survey instrument able to map large areas of sky with unprecedented survey speed and sensitivity.

The large data stream produced by ASKAP will be processed and archived at the Pawsey High-Performance Computing Centre for SKA Science based in Perth. The Pawsey Centre was established in 2009 as part of the Australian Government's Super Science initiative with the aim to process data from ASKAP, the MWA, and ultimately the SKA. The ASKAP data stream will be processed in real time with the help of so-called "software instruments", which will be optimised for specific observing modes. After processing and quality control, the data will be archived at the Pawsey Centre where it can be accessed by astronomers from around the world. There will be no proprietary period for ASKAP data, and all processed data products, including images and catalogues, will be made publicly available after a short period of quality control.

Construction of ASKAP commenced with the delivery of the first antenna at the end of 2009, and all 36 antennas - built by the $54^{\text {th }}$ Research Institute of China Electronics Technology Group Corporation (CETC54) in Shijiazhuang, China - and their technical systems will be completed in 2013. Once built, ASKAP will operate as part of CSIRO's radio-astronomy facility for use by Australian and international scientists. As well as being a world-leading telescope in its own right, ASKAP will be an important test-bed for the SKA.

\footnotetext{
${ }^{1}$ http://www.atnf.csiro.au/projects/askap/config.html
} 

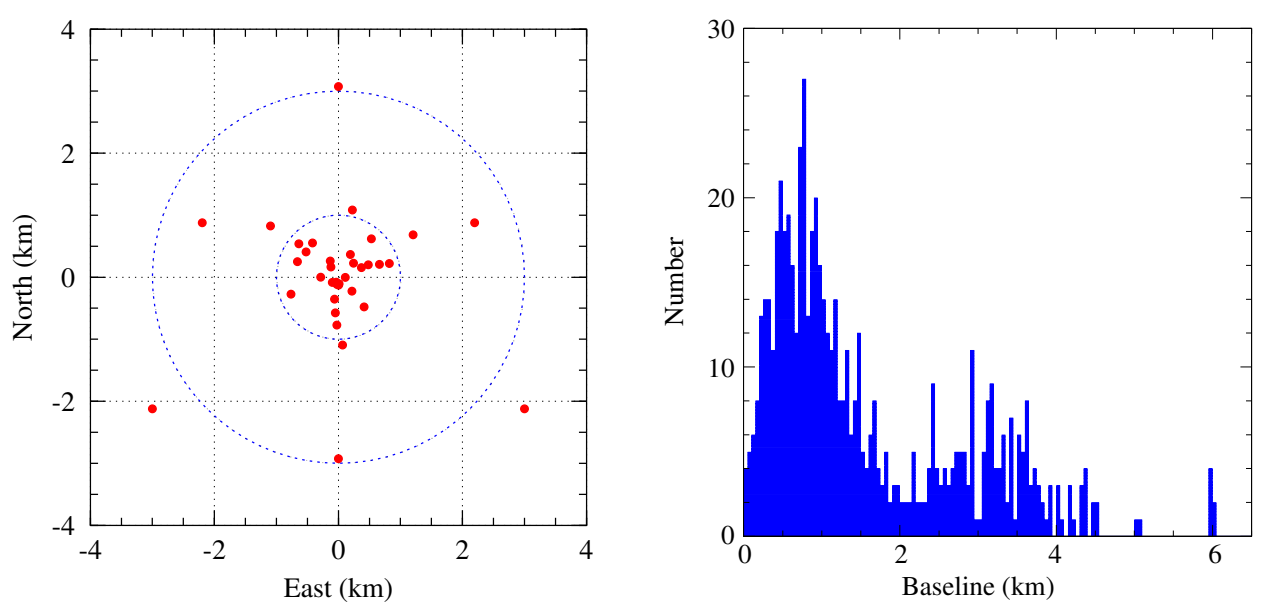

Figure 2: Left: Layout of the 36 antennas of the initial ASKAP configuration (red dots). The blue circles have diameters of 2 and $6 \mathrm{~km}$, respectively. Right: Histogram of telescope baseline lengths for the initial ASKAP configuration.

\section{ASKAP Survey Science Projects}

During ASKAP's first five years of operation, about $75 \%$ of its time will be used for large Survey Science Projects which require more than 1500 hours of observing time and make use of ASKAP's unique capabilities and survey speed (see [5] and [6] for a detailed outline of the science case for ASKAP). The remaining 25\% of the time will be dedicated to smaller Guest Science Projects of less than 1500 hours.

An open, international, and competitive process was set up for submission of expressions of interest and proposals for large Survey Science Projects. An international panel of expert astronomers with a broad range of proficiencies picked the projects that will take advantage of ASKAP's survey speed and large field-of-view.

Based on the recommendations of the review panel, CSIRO announced in September 2009 that ten major Survey Science Projects, representing 363 unique authors from 131 institutions all across the world, had been selected to use ASKAP in its first five years of operation. These projects will address widely recognised astrophysical issues, such as the evolution of star-forming galaxies and massive black holes, variable and transient radio sources, the interstellar medium of our own Galaxy, magnetic fields in the Milky Way and other galaxies, and pulsars. Their results will be important for the broad astronomical community.

Of the ten projects' authors, 33\% are from Australia and New Zealand, 30\% from North America, 28\% from Europe, and 9\% from elsewhere in the world. Principal Investigators and team members of the ASKAP Survey Science Projects were invited to work with CSIRO through the design study phase. Members of the project teams, together with the ASKAP Project Scientists and other ASKAP staff, have formed ASKAP working groups that meet on a regular basis to ensure close collaboration between CSIRO and the Survey Science Projects.

The ten ASKAP Survey Science Project proposals selected by the international committee were prioritised into three categories: an "A" group, for which CSIRO will provide full support (EMU and WALLABY), an "A-" group, for which CSIRO will make all reasonable efforts to 

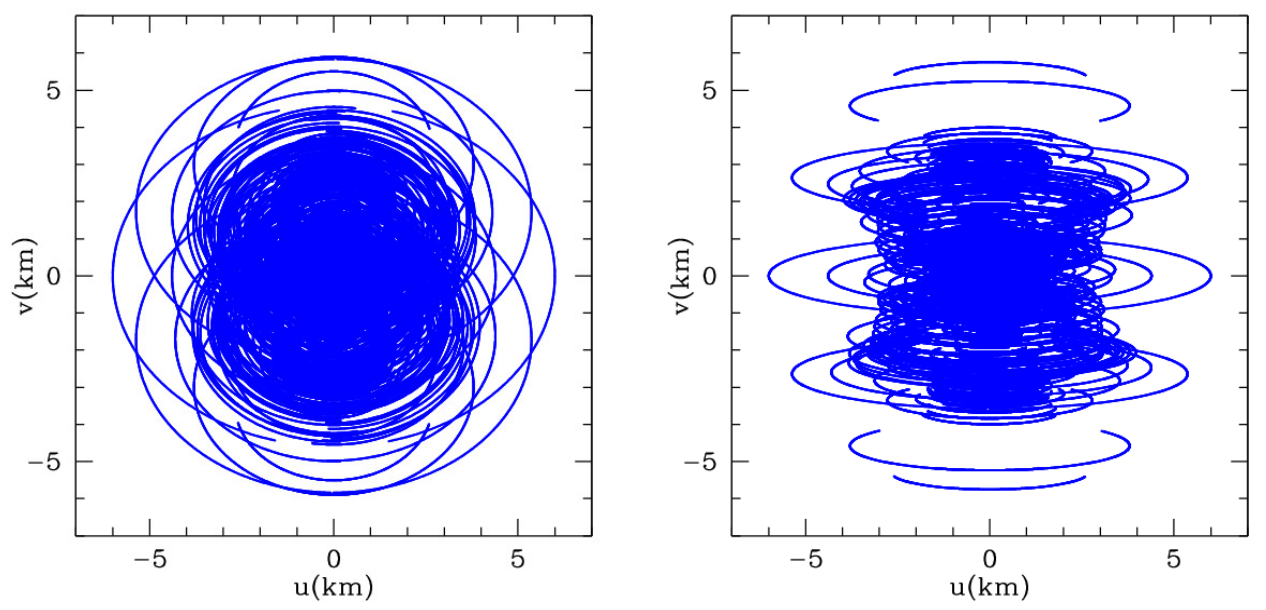

Figure 3: $u v$ coverage of ASKAP for declinations of $-50^{\circ}$ (left) and $-10^{\circ}$ (right) and a total integration time of $10 \mathrm{~h}$ (hour-angle coverage: $\pm 5 \mathrm{~h}$ around transit).

support (FLASH, VAST, GASKAP, POSSUM, CRAFT, and DINGO), and a "Strategic Priorities" group (VLBI and COAST), for which CSIRO will work to ensure that capabilities are enabled to the extent possible. The ten Survey Science Projects and their main scientific aims are outlined in more detail in the following pages.

\subsection{EMU: Evolutionary Map of the Universe}

Principal Investigator: Ray Norris (CSIRO, Australia)

EMU is a deep ( $10 \mu \mathrm{Jy} /$ beam rms) radio continuum survey of $75 \%$ of the entire sky [7]. EMU will probe typical star forming galaxies to redshift 1, powerful starbursts to even greater redshifts, Active Galactic Nuclei to the edge of the Universe, as well as undoubtedly discovering new classes of rare objects. The key science goals for EMU are to trace the evolution of star forming galaxies and massive black holes throughout the history of the Universe and to explore large-scale structure. EMU will create the most sensitive wide-field atlas yet made and provide a long-lasting legacy survey.

\subsection{WALLABY: Widefield ASKAP L-band Legacy All-sky Blind Survey}

Principal Investigators: Bärbel Koribalski (CSIRO, Australia) and Lister StaveleySmith (ICRAR, Australia)

WALLABY is an extragalactic neutral hydrogen survey over $75 \%$ of the entire sky and will detect up to 500,000 galaxies to a redshift of 0.26. The fundamental aims of WALLABY are to examine the H I properties and large-scale distribution of these galaxies in order to study galaxy formation and the missing satellite problem in the Local Group, evolution and star formation of galaxies, the role of mergers and galaxy interactions, the H I mass function and its variation with galaxy density, the physical processes governing the distribution and evolution of cool gas at low redshift, cosmological parameters relating to gas-rich galaxies, and the nature of the cosmic web. WALLABY will provide the largest, most homogeneous H I sample of galaxies yet made, and will be an important pathfinder for key SKA science. 


\subsection{FLASH: First Large Absorption Survey in H I}

Principal Investigator: Elaine Sadler (The University of Sydney, Australia)

FLASH is a blind H I absorption-line survey that uses background radio continuum sources to identify and characterise foreground neutral hydrogen. FLASH science outcomes are focused on both the neutral gas content of galaxies and the cosmic H I mass density in the redshift range $0.5<z<1.0$ where the H I emission line is too weak to be detectable in individual galaxies. The observations will increase the total number of absorption line systems by an estimated two orders of magnitude, representing a significant data set to study gas assembly and galaxy formation during a time in the history of the Universe that is largely unstudied thus far.

\subsection{VAST: An ASKAP Survey for Variables and Slow Transients}

Principal Investigators: Tara Murphy (The University of Sydney, Australia) and Shami Chatterjee (Cornell University, USA)

VAST gives unprecedented opportunities to investigate the sky at radio wavelengths for transients with a timescale as short as 5 seconds. ASKAP's wide-field survey capabilities will enable the discovery and investigation of variable and transient phenomena from the local to the cosmological including flare stars, intermittent pulsars, X-ray binaries, magnetars, extreme scattering events, intra-day variables, radio supernovae and the orphan afterglows of gamma-ray bursts. VAST will probe unexplored regions of phase space where new classes of transient sources may be detected.

\subsection{GASKAP: The Galactic ASKAP Spectral Line Survey}

Principal Investigators: John Dickey (University of Tasmania, Australia) and Naomi McClure-Griffiths (CSIRO, Australia)

GASKAP is a high spectral resolution survey of the H I and OH lines in the Milky Way and the Magellanic system. Compared with existing data, GASKAP will achieve about an order of magnitude improvement in both brightness sensitivity and angular resolution. GASKAP will detect and map $\mathrm{OH}$ masers from evolved stars and star formation regions, diffuse emission from molecular and atomic clouds, H I absorption toward continuum sources, and structures in the gas that trace the effects of stellar winds and supernova explosions. The Magellanic Clouds will show all these processes as they appear in two other, very different environments. GASKAP will provide stunning images of the ISM that will be indispensable for astronomers working at other wavelengths.

\subsection{POSSUM: Polarisation Sky Survey of the Universe's Magnetism}

Principal Investigators: Bryan Gaensler (The University of Sydney, Australia), Russ

Taylor (University of Calgary, Canada), and Tom Landecker (DRAO, Canada)

Understanding the Universe is impossible without understanding magnetic fields. Magnetic fields are key to the non-thermal Universe, yet it is unclear how large-scale magnetic fields are generated and maintained. POSSUM will use radio source polarisation, in particular the technique of rotation measure (RM) synthesis, to perform a wide-field survey that will yield a grid of RMs 
over a substantial fraction of the sky. The science outcomes of POSSUM will revolutionise our understanding of the ordered components of the Milky Way's magnetic field, test dynamo and other models of magnetic field generation in galaxies and clusters, and carry out a comprehensive census of magnetic fields as a function of redshift in galaxies, active galactic nuclei, galaxy clusters, and the intergalactic medium.

\subsection{CRAFT: The Commensal Real-time ASKAP Fast Transients Survey}

Principal Investigator: Peter Hall (ICRAR, Australia)

CRAFT is a purely commensal survey for transient sources with timescales shorter than 5 seconds [8]. Short-timescale transients are associated with the most energetic and brightest single events in the Universe. They provide nature's ultimate laboratory; their emission is generated by matter under extreme conditions whose properties probe physical regimes far transcending the range achievable in terrestrial experiments. Fast timescale transients open new vistas on the physics of high brightness temperature objects, extreme states of matter, and the physics of strong gravitational fields. In addition, the detection of extragalactic transients affords us an entirely new and sensitive probe on the huge reservoir of baryons in the intergalactic medium.

\subsection{DINGO: Deep Investigation of Neutral Gas Origins}

\section{Principal Investigator: Martin Meyer (ICRAR, Australia)}

DINGO will study the evolution of neutral hydrogen (H I) from the current epoch to a redshift of about 0.5 , providing a legacy dataset spanning cosmologically representative volumes [9]. DINGO will measure key cosmological distributions, including $\Omega_{\mathrm{HI}}$, the $\mathrm{HI}$ mass function, and the halo occupation distribution function. ASKAP data will be combined with optical data to enable a thorough study of the co-evolution of the stellar, baryonic, and dark matter content of galaxies.

\subsection{VLBI: The High-resolution Components of ASKAP - Meeting the Long Baseline Specifications for the SKA}

Principal Investigator: Steven Tingay (ICRAR, Australia)

ASKAP, in combination with the existing Australian Long Baseline Array, high-speed data recording equipment, innovative software correlation facilities, and high-speed data transport networks, provides a high-resolution capability that is unmatched in terms of SKA demonstrators around the world. Science outcomes include proper motion and parallax of pulsars, high-resolution imaging of Active Galactic Nuclei, follow-up of transient radio sources, and distances and proper motions of $\mathrm{OH}$ masers.

\subsection{COAST: Compact Objects with ASKAP - Surveys and Timing}

Principal Investigator: Ingrid Stairs (The University of British Columbia, Canada)

COAST will undertake an observational program of pulsar timing aimed at high-profile issues in astrophysics. This includes limits on, or the detection of, a background of gravitational waves, 

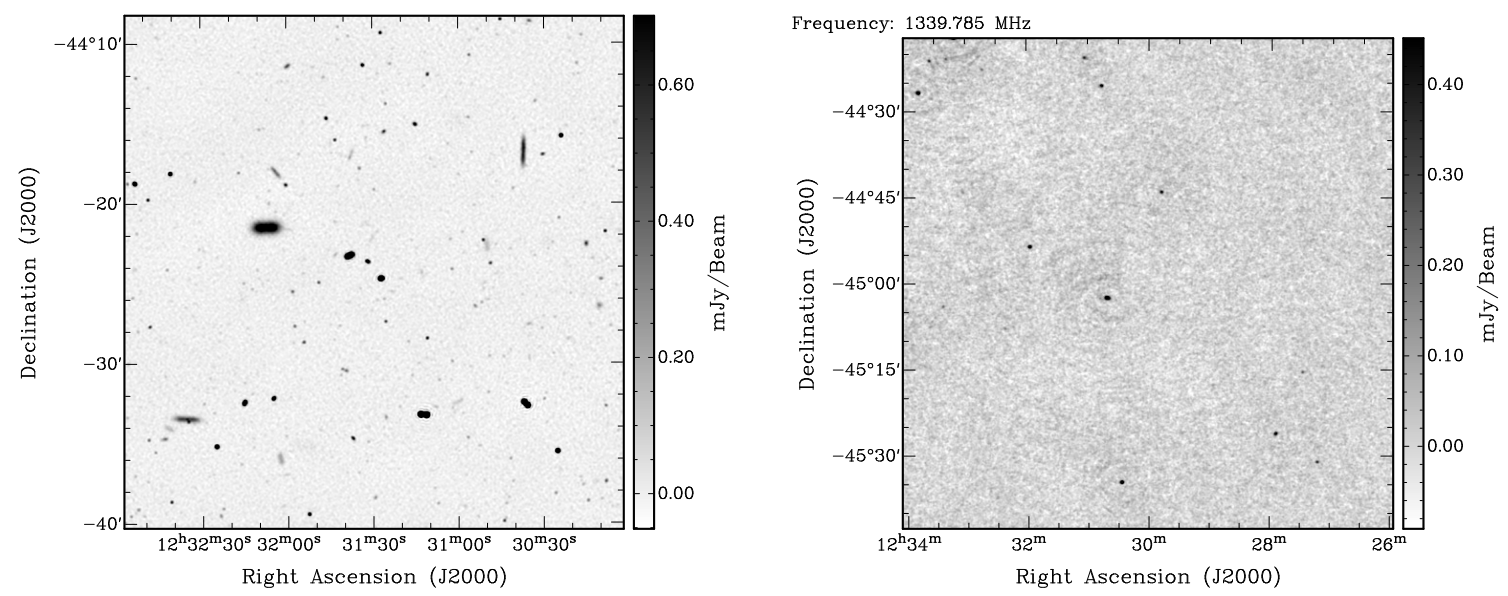

Figure 4: Left: Detail of the most recent ASKAP continuum simulation ( $8 \mathrm{~h}$ integration, $10^{\prime \prime}$ resolution, Stokes I) released in May 2010. Right: Detail of the most recent ASKAP spectral-line simulation (single channel, $8 \mathrm{~h}$ integration, 30" resolution, artificially reduced noise) released in April 2010.

stringent tests of the predictions of General Relativity and other theories of strong gravity, and the studies of binary stellar evolution. In addition to pulsar timing, blind searches for pulsars will also be carried out which will lead to a better understanding of the Galactic neutron star population, the pulsar emission mechanism, and the structure and magnetic field of the Galaxy.

\section{ASKAP Simulations}

End-to-end simulations of ASKAP data are released on a regular basis as part of our work on the ASKAP telescope simulator and imaging pipeline. The purpose of these simulations is to

- develop the software pipelines required for reducing and processing ASKAP data,

- understand the instrumental and observational effects on the imaging process, and

- provide the Survey Science Teams with simulated ASKAP data for the purpose of developing source finding and data analysis strategies.

The simulations are based on the SKADS S3-SAX sky model [10] for the H I sky and the SKADS S3-SEX sky model [11] for the radio continuum sky. The sky models are then passed through a simulation of the telescope and subsequently imaged. The code for the imaging and telescope simulation, developed in-house specifically for ASKAP data processing, is ASKAPSOFT which uses the CASACORE library but has extra functionality.

The ASKAP team has a copy of the SKADS skies as developed by the group at Oxford University. The continuum sky covers an area of $20^{\circ} \times 20^{\circ}$ with approximately 250 million sources down a flux limit of $10 \mathrm{nJy}$. Sources have total intensity fluxes only (no polarisation). The H I data consist of a catalogue of 43 million galaxies out to a redshift of 1.06, covering an area of $11.4^{\circ} \times 11.4^{\circ}$ on the sky. The catalogue is reasonably complete above $10^{8} M_{\odot}$. The emission spectra are characterised by 5 parameters, but essentially all are symmetrical, double-horned spectra. 


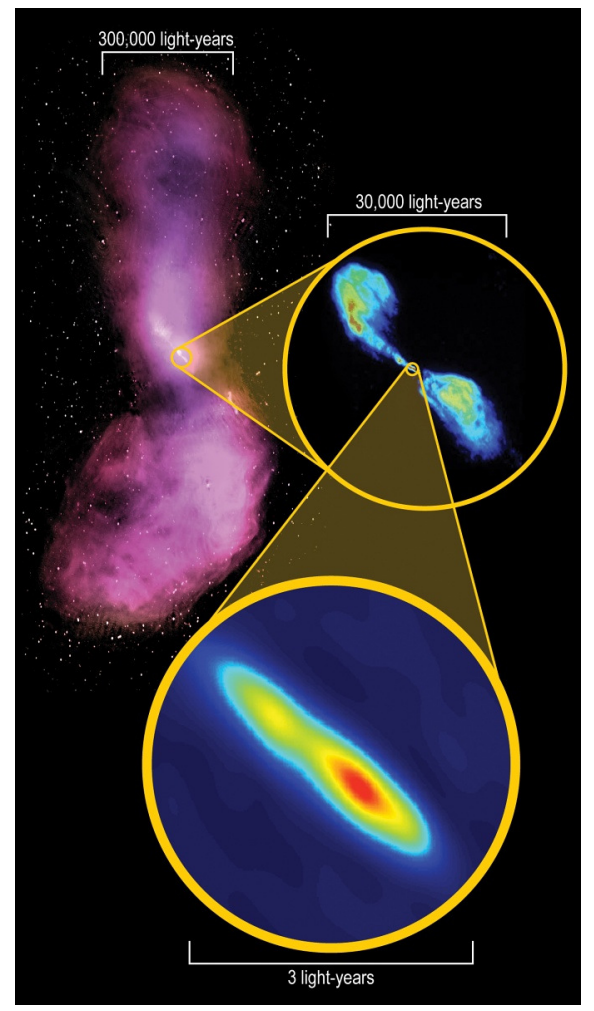

Figure 5: Centaurus A in the CSIRO media release of May 26, 2010. Top-left: $21-\mathrm{cm}$ radio continuum image obtained with the Australia Telescope Compact Array and the 64-m Parkes telescope (I. Feain, T. Cornwell \& R. Ekers, CSIRO; R. Morganti, ASTRON; N. Junkes, MPIfR). Top-right: 6-cm radio continuum image obtained with the Very Large Array (NRAO / AUI / NSF). Bottom: 21-cm radio continuum image obtained with the Long Baseline Array in combination with the first ASKAP antenna and the 12-m Warkworth telescope (S. Tingay, ICRAR; ICRAR, CSIRO and AUT).

There is no continuum associated with the line-emitting galaxies. More details and a discussion of the limitations of these models can be found in the original papers by [10] and [11].

The left-hand panel of Fig. 6 shows a detail of the most recent continuum simulation at $10^{\prime \prime}$ resolution, using the entire array of 36 antennas. The right-hand panel of Fig. 7 shows a detail of a single frequency channel of the most recent spectral-line simulation. The noise level in this particular data set was artificially reduced to increase the number of detectable H I sources for the purpose of source finder testing.

In addition to these basic continuum and spectral-line data sets, simulations including transient and polarised continuum sources have been released to assist the VAST and POSSUM teams in their development of source finding and data analysis strategies. Future simulations will cover areas such as diffuse continuum and $\mathrm{HI}$ emission, combined continuum and spectral line simulations, more sophisticated simulations of transient and polarised sources, inclusion of observational and instrumental errors in the simulations, etc. All simulations have been distributed to the Survey Science Teams via ASKAP's internal project management website. They have also been made publicly available on the internet. ${ }^{2}$

\section{Recent Developments and Outlook}

The first ASKAP antenna, named "Diggidumble" by the local Aboriginal community, has been up and running at the MRO site since the end of 2009 (left-hand image in Fig. 1). The foundations

\footnotetext{
${ }^{2}$ http://www.atnf.csiro.au/people/Matthew. Whiting/ASKAPsimulations.php
} 
for antennas 2 to 6 were poured at the end of May 2010, with the antennas scheduled to arrive on site in early September 2010. These first six antennas will form the so-called Boolardy Engineering Test Array (BETA) which will be used for extensive testing of the entire system, including the antenna hardware, phased array feeds, correlators, observing and calibration techniques, and data reduction pipelines. The first results from synthesis observations with BETA are anticipated for $\operatorname{mid} 2011$.

In April 2010, the first science observations involving ASKAP were carried out as part of VLBI observations with the Australian Long Baseline Array (LBA) [12] in combination with the first ASKAP antenna and the new 12-m radio telescope operated by Auckland University of Technology near Warkworth on New Zealand's North Island. The successful observations targeted the nucleus of Centaurus A as well as the radio galaxy PKS 1934-638 at $1.4 \mathrm{GHz}$ with unprecedented angular resolution as compared to the classic LBA. Fig. 5 shows the image of Centaurus A which accompanied a CSIRO media release on May 26, 2010.

The Australian Minister for Innovation, Industry, Science and Research, Senator Kim Carr, announced the outcome of the Australian Government's Super Science Fellowship programme during a ceremony held on April 8, 2010. The government will provide a total of AUD 27.2 million of funding for two rounds of three-year, early-career research fellowships in three areas of research. Space science and astronomy, one of the three supported research areas, will receive funds for a total of 33 Super Science Fellows over the next four years. About ten of these fellowships are directly related to ASKAP or one of the Survey Science Projects.

Furthermore, CSIRO's "Sustainable Energy for SKA" bid to the Sustainability Round of the Education Investment Fund of the Australian Government has been successful. On June 9, 2010 the Australian Prime Minister, Kevin Rudd, made the announcement of the AUD 47.3 million award for the development of renewable energy provisions for both the Pawsey Centre in Perth as well as the Murchison Radio-astronomy Observatory.

\section{Further Information}

More information on ASKAP is available from the official ASKAP website. ${ }^{3}$ In addition, ASKAP Science Updates ${ }^{4}$ and ASKAP Technical Updates ${ }^{5}$ are being published in regular intervals to report on the latest developments and activities in the areas of science and engineering. The Australia and New Zealand SKA Project (anzSKA) has its own website ${ }^{6}$ with comprehensive information on the SKA activities in Australia and New Zealand, including the regular anzSKA Newsletter. ${ }^{7}$ For additional information and enquiries about ASKAP please contact the ASKAP Project Scientists, Simon Johnston and Ilana Feain. ${ }^{8}$

\footnotetext{
${ }^{3}$ http: //www.atnf.csiro.au/projects/askap/

${ }^{4}$ http://www.atnf.csiro.au/projects/askap/science_update_newsletter.html

5 http://www.atnf.csiro.au/projects/askap/technical_update_newsletter.html

${ }^{6}$ http://www.ska.gov.au/

${ }^{7}$ http://www.ska.gov.au/media/newsletter/

${ }^{8}$ E-mail address: atnf-askap-psecsiro.au
} 


\section{Acknowledgments}

We acknowledge the Wajarri Yamatji people as the traditional owners of the Murchison Radioastronomy Observatory site.

\section{References}

[1] D. R. DeBoer, R. G. Gough, J. D. Bunton, T. J. Cornwell, R. J. Beresford, et al., 2009, Australian SKA Pathfinder: A High-Dynamic Range Wide-Field of View Survey Telescope, Proceedings of the IEEE, 97,1507

[2] P. E. Dewdney, P. J. Hall, R. T. Schilizzi, \& T. J. L. W. Lazio, 2009, The Square Kilometre Array, Proceedings of the IEEE, 97, 1482

[3] C. J. Lonsdale, R. J. Cappallo, M. F. Morales, F. H. Briggs, L. Benkevitch, et al., 2009, The Murchison Widefield Array: Design Overview, Proceedings of the IEEE, 97, 1497

[4] N. Gupta, S. Johnston, I. Feain, \& T. Cornwell, 2008, The Initial Array Configuration for ASKAP, ATNF ASKAP Memo Series, 21, available at http://www . atnf.csiro.au/projects / askap/Memoseries.html

[5] S. Johnston, R. Taylor, M. Bailes, N. Bartel, C. Baugh, et al., 2008, Science with ASKAP - The Australian Square-Kilometre-Array Pathfinder, ExA, 22, 151

[6] S. Johnston, M. Bailes, N. Bartel, C. Baugh, M. Bietenholz, et al., 2007, Science with the Australian Square Kilometre Array Pathfinder, PASA, 24, 174

[7] R. P. Norris \& the EMU team, 2009, ASKAP-EMU: Overcoming the challenges of wide deep continuum surveys, in proceedings of Panoramic Radio Astronomy: Wide-field 1-2 GHz research on galaxy evolution, June 2-5, 2009, Groningen, the Netherlands, POS (PRA2009) 033

[8] J.-P. Macquart \& the CRAFT collaboration, 2010, The Commensal Real-time ASKAP Fast Transients (CRAFT) survey, PASA, 27, 272

[9] M. Meyer \& the DINGO team, 2009, Exploring the HI Universe with ASKAP, in proceedings of Panoramic Radio Astronomy: Wide-field 1-2 GHz research on galaxy evolution, June 2-5, 2009, Groningen, the Netherlands, PoS (PRA2009) 015

[10] D. Obreschkow, H.-R. Klöckner, I. Heywood, F. Levrier, \& S. Rawlings, 2009, A Virtual Sky with Extragalactic HI and CO Lines for the Square Kilometre Array and the Atacama Large Millimetre/Submillimetre Array, ApJ, 703, 1890

[11] R. I. Wilman, L. Miller, M. J. Jarvis, T. Mauch, F. Levrier, et al., 2008, A semi-empirical simulation of the extragalactic radio continuum sky for next generation radio telescopes, MNRAS, 388, 1335

[12] R. P. Norris, 1988, The Australia Telescope Long Baseline Array, in proceedings of The Impact of VLBI on Astrophysics and Geophysics, 129 ${ }^{\text {th }}$ IAU Symposium, May 10-15, 1987, Cambridge, MA, USA 